\title{
Wear problem for tube with thin nonuniform inner coating and rigid insert with complex profile
}

\author{
Kirill E Kazakov ${ }^{1, *}$, Svetlana P Kurdina ${ }^{2}$ \\ ${ }^{1}$ Ishlinsky Institute for Problems in Mechanics RAS, 119526, pr-t Vernadskogo 101-1, Moscow, Russia \\ ${ }^{2}$ Bauman Moscow State Technical University, 105005, 2ya Baumanskaya 5-1, Moscow, Russia
}

\begin{abstract}
The article describes the formulation of the problem of wear of an elastic pipe with a nonuniform internal coating by a rigid insert, the diameter of which is variable. For this problem, a mathematical model is built, which is a mixed integral equation. The resulting integral equation contains functions related to the variable properties of the coating and the shape of the insert. When constructing an analytical solution, a special approach was used, since standard methods do not allow taking into account the presence of rapidly changing functions in the equation, which can describe the shapes and properties of materials. In the resulting solution, the functions describing the variable properties of the coating and the profile of the insertion are separated by separate terms. This allows you to always get accurate results.
\end{abstract}

\section{Introduction}

Pipes are widely used in industry. Both internal and external coatings can be used to protect pipes from the effects of aggressive media, for thermal insulation or electrical insulation. Due to the peculiarities of the application of such coatings, they can have nonuniformity. Often, rigid inserts are used to connect various sections or reinforce sections. Due to the vibrations of the inserts, pipe wear occurs.

A preliminary calculation of the wear-contact interaction between the pipe and the liner allows predicting the distribution of stresses and deformations in such bodies, as well as estimating the amount of pipe wear. Such studies will allow engineers to take the necessary steps at the design stage to modify the design or its operating conditions, which will lead to future economic benefits.

The paper considers the problem of linear fretting wear of an inner thin nonuniform layer of a pipe interacting with a rigid insert with a variable diameter. The construction of the solution is based on the results of earlier works devoted to the study of the wear of coated flat bases [1-3] and pipes with an external coating [4, 5]. It should be noted that mathematical model works only for the case of small wear compared coating thickness.

\section{Linear mathematical model of wear contact problem}

The long elastic pipe consists of an external layer of the thickness $h_{\text {out }}$ and an thin elastic nonuniform layer of the thickness $h_{\text {in }}$ (Fig. 1). Nonuniformity of a layer is understood as the dependence of its elastic properties on the longitudinal coordinate $z$ (Young's modulus $E_{\text {in }}(z)$ and Poisson's ratio $\left.v_{\text {in }}(z)\right)$. The axis $z$ is directed along the axis of the pipe. There is a smooth contact between these layers.

At a predetermined time $\tau_{0}$, an insert is placed inside the above-described pipe. The variable outer radius of the insert $g(z)$ is greater than the inner radius of the pipe $r_{\text {in }}-h_{\text {in. }}$. Due to this fact, the pipe is deformed and stresses arise in it. There is a smooth contact along $z$-axis between inner layer and insert. Moreover due to the vibrations of the inserts coating begins to wear out. It is necessary to determine the distribution of the contact stresses in tube in the area of the insert in time.

The following assumptions are made: the coating thickness $h_{\text {in }}$ is much less than the insert length $2 a$, the outer layer inner radius $r_{\text {in }}$, and the outer layer thickness $h_{\text {out }}$; the inner layer stiffness is less than the outer layer stiffness; the amount of wear for all considered times is small compared to the thickness of the coating $h_{\text {in }}$.

To construct the mathematical model of the problem, the rigid insert is replaced by a some load $q(z, t)$ acting on region in contact region $(z \in[-a, a])$, and equal to zero outside this region. Displacement of inner boundary of coating is equal to sum of displacement due to load and displacement due to wear.

A number of experiments show that the wear velocity linearly depends on the relative average velocity $V$ of the contacting surfaces and on the normal load $q(z, t)$ and is inversely proportional to the hardness $H(z)$ of the material [6-10]:

Corresponding author: kazakov-ke@,yandex.ru 


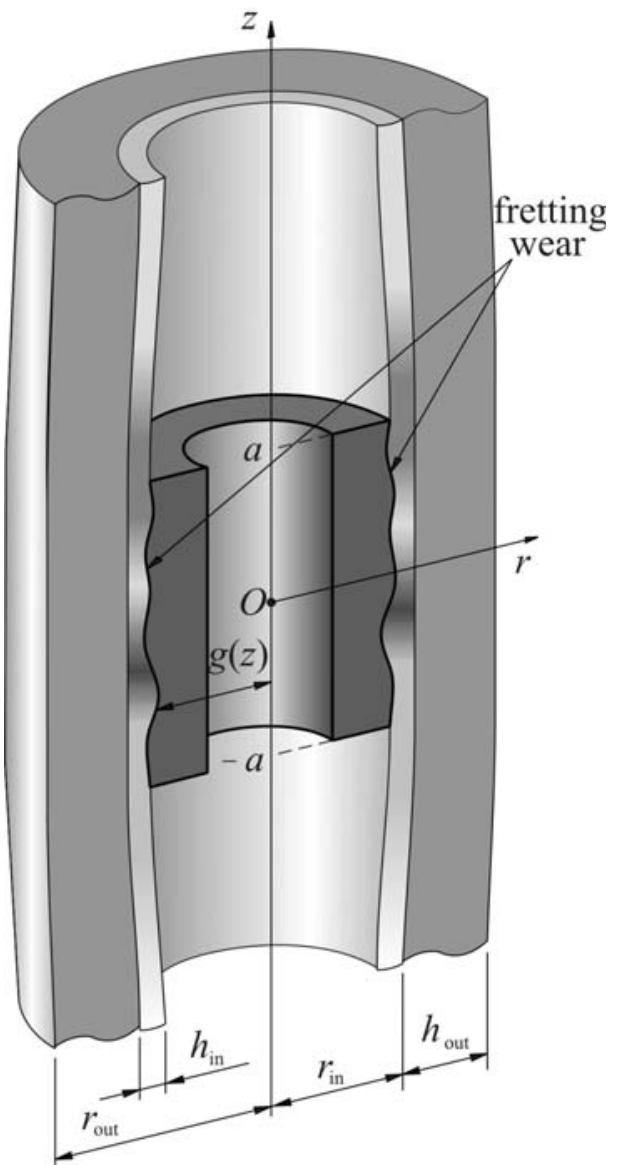

Fig. 1. Wear of elastic tube with coating.

$$
v_{\mathrm{w}}(z, t)=\frac{k_{\mathrm{w}} V q(z, t)}{H(z)},
$$

where $k_{\mathrm{w}}$ is experimentally determined constant. The velocity $V$ can be estimated with fretting-wear of surfaces, when the angular velocity is absent, however, the coating wears out due to vibrations of one body relative to another.

Then the vertical displacement of outer surface due to wear can be determined by integrating of wear velocity $v_{\mathrm{w}}(z, t)$ over time from start moment until current time

$$
u_{\mathrm{w}}(z, t)=\int_{\tau_{0}}^{t} v_{\mathrm{w}}(z, \tau) d \tau=\frac{k_{\mathrm{w}} V}{H(z)} \int_{\tau_{0}}^{t} q(z, \tau) d \tau .
$$

It can be shown that expressions for displacements of outer layer of tube with nonuniform coating due to load $q(z, t)$ almost coincide with expressions for the case of uniform layers (see, for example, [11, 12]). Generalization of the results of these works for the case when the coating is nonuniform and the above assumptions are fulfilled, makes it possible to construct the following mixed integral equation, in which the unknown function is the distribution of contact stresses

$$
\begin{aligned}
& u_{\mathrm{q}}(z, t)=\frac{1-v_{\text {in }}^{2}(z)}{E_{\text {in }}(z)} h_{\text {in }} q(z, t) \\
& +\frac{2\left(1-v_{\text {out }}^{2}\right)}{\pi E_{\text {out }}} \int_{-a}^{a} k_{\mathrm{c}}\left(\frac{z-\zeta}{r_{\text {in }}}\right) q(\zeta, t) d \zeta .
\end{aligned}
$$

Here $E_{\text {out }}$ and $v_{\text {out }}$ are Young's modulus and Poisson's ratio of outer layer, $k_{\mathrm{c}}(s)$ is known kernel of the cylindrical contact problem

$$
k(s)=\int_{0}^{\infty} \frac{L(u)}{u} \cos (s u) d u,
$$

where

$$
\begin{gathered}
L(u)=\frac{u\left[f\left(k_{r}, u\right) D_{1}^{2}(u)+k_{r}^{-1}-k_{r} u^{2} C_{1}^{2}(u)\right]}{S(u)}, \\
f(r, u)=\frac{2\left(1-v_{\text {in }}\right)}{r}+u^{2} r, k_{r}=\frac{r_{\text {out }}}{r_{\text {in }}}, \\
S(u)=\frac{f(1, u)}{k_{r}}+f\left(k_{r}, u\right)+k_{r} u^{4} A_{1}^{2}(u)-u^{2} f\left(k_{r} u\right) B_{1}^{2}(u) \\
-k_{r} u^{2} f(1, u) C_{1}^{2}(u)+f(1, u) f\left(k_{r}, u\right) D_{1}^{2}(u), \\
A_{1}(u)=\mathrm{I}_{0}(u) \mathrm{K}_{0}\left(k_{r} u\right)-\mathrm{I}_{0}\left(k_{r} u\right) \mathrm{K}_{0}(u), \\
B_{1}(u)=\mathrm{I}_{0}(u) \mathrm{K}_{1}\left(k_{r} u\right)+\mathrm{I}_{1}\left(k_{r} u\right) \mathrm{K}_{0}(u), \\
C_{1}(u)=\mathrm{I}_{0}\left(k_{r} u\right) \mathrm{K}_{1}(u)+\mathrm{I}_{1}(u) \mathrm{K}_{0}\left(k_{r} u\right), \\
D_{1}(u)=\mathrm{I}_{1}(u) \mathrm{K}_{1}\left(k_{r} u\right)-\mathrm{I}_{1}\left(k_{r} u\right) \mathrm{K}_{1}(u),
\end{gathered}
$$

$\mathrm{I}_{0}(u), \mathrm{I}_{1}(u), \mathrm{K}_{0}(u), \mathrm{K}_{1}(u)$ are modified Bessel functions of first and second kind, $r_{\text {out }}$ is the outer radius of the outer layer in undeformed state.

Using equations (2) and (3) one can obtain radial displacement of inner surface of layered tube due to wear and distributed load (it is assumed that amount of wear $u_{\mathrm{w}}(z, t)$ is small compared to coating thickness $\left.h_{\text {in }}\right)$

$$
\begin{gathered}
u_{\text {in }}(z, t)=\frac{1-v_{\text {in }}^{2}(z)}{E_{\text {in }}(z)} h_{\text {in }} q(z, t)+\frac{k_{\mathrm{w}} V}{H(z)} \int_{\tau_{0}}^{t} q(z, \tau) d \tau \\
+\frac{2\left(1-v_{\text {out }}^{2}\right)}{\pi E_{\text {out }}} \int_{-a}^{a} k_{\mathrm{c}}\left(\frac{z-\zeta}{r_{\text {in }}}\right) q(\zeta, t) d \zeta .
\end{gathered}
$$

On the other hand, the movement of the inner surface $u_{\text {in }}(z, t)$ is known: it is equal to the difference between the insert radius $g(z)$ and the inner diameter of the inner pipe at the current moment, that is

$$
\begin{aligned}
u_{\text {in }}(z, t) & =g(z)-\left[r_{\text {in }}-\left(h_{\text {in }}-u_{\mathrm{w}}(z, t)\right)\right] \\
= & g(z)-r_{\text {in }}+h_{\text {in }}-u_{\mathrm{w}}(z, t) .
\end{aligned}
$$

Equating the right-hand sides of formulas (6) and (7), you can get the following mixed integral equation:

$$
\begin{gathered}
\frac{1-v_{\text {in }}^{2}(z)}{E_{\text {in }}(z)} h_{\text {in }} q(z, t)+2 \frac{k_{\mathrm{w}} V}{H(z)} \int_{\tau_{0}}^{t} q(z, \tau) d \tau \\
+\frac{2\left(1-v_{\text {out }}^{2}\right)}{\pi E_{\text {out }}} \int_{-a}^{a} k_{\mathrm{c}}\left(\frac{z-\zeta}{r_{\text {in }}}\right) q(\zeta, t) d \zeta \\
=g(z)-r_{\text {in }}+h_{\text {in }} .
\end{gathered}
$$

In some cases, the hardness and stiffness of materials can be proportional (see, for example, $[13,14])$. In this paper, this particular case is considered, therefore

$$
H(z)=k_{\mathrm{H}} R(z) \text {. }
$$

Here $k_{\mathrm{H}}$ is known coefficient, $R(z)$ is contact rigidity of inner coating equal to

$$
R(z)=\frac{E_{\text {in }}(z)}{1-v_{\text {in }}^{2}(z)} .
$$

Previously, similar assumptions were used in papers [15]. Thus, taking into account assumption (9), equation (8) takes the form 


$$
\begin{gathered}
\frac{h_{\text {in }}}{R(z)}\left[q(z, t)+\frac{2 k_{\mathrm{w}} V}{k_{\mathrm{H}} h_{\text {in }}} \int_{\tau_{0}}^{t} q(z, \tau) d \tau\right] \\
+\frac{2\left(1-v_{\text {out }}^{2}\right)}{\pi E_{\text {out }}} \int_{-a}^{a} k_{\mathrm{c}}\left(\frac{z-\zeta}{r_{\text {in }}}\right) q(\zeta, t) d \zeta \\
=g(z)-r_{\text {in }}+h_{\text {in }} .
\end{gathered}
$$

Note that function $R(z)$ describes elastic properties of inner coating. It can be rapidly changing or discontinuous.

The problem is to find the contact stresses $q(z, t)$ from equation (11).

\section{Dimensionless form of mixed integral equation}

The resulting mixed integral equation (11) can be reduced to a dimensionless form. To do this, the following change of variables is performed

$$
\begin{gathered}
z^{*}=\frac{z}{a}, \quad \zeta^{*}=\frac{\zeta}{a}, \quad t^{*}=\frac{t}{\tau_{0}}, \quad \delta^{*}=\frac{\delta}{a}, \\
g^{*}\left(z^{*}\right)=\frac{r_{\text {in }}-h_{\text {in }}-g(z)}{a}, \\
m^{*}\left(z^{*}\right)=\frac{h_{\text {in }} E_{\text {out }}}{2\left(1-v_{\text {out }}^{2}\right) a R(z)}, \\
q^{*}\left(z^{*}, t^{*}\right)=\frac{2\left(1-v_{\text {out }}^{2}\right) q(z, t)}{E_{\text {out }}}, \\
K^{*}\left(t^{*}, \tau^{*}\right)=-\frac{2 k_{\mathrm{w}} V \tau_{0}}{k_{\mathrm{H}} h_{\text {in }}}=-V^{*}=\text { const, } \\
k^{*}\left(z^{*}, \zeta^{*}\right)=\frac{1}{\pi} k_{\mathrm{c}}\left(\frac{z-\zeta}{r_{\text {in }}}\right) \\
\mathbf{V}^{*} f\left(t^{*}\right)=\int_{1}^{t^{*}} K\left(t^{*}, \tau^{*}\right) f\left(\tau^{*}\right) d \tau^{*}, \\
\mathbf{F}^{*} f\left(z^{*}\right)=\int_{-1}^{1} k^{*}\left(z^{*}, \zeta^{*}\right) f\left(\zeta^{*}\right) d \zeta^{*}
\end{gathered}
$$

With this change, the mixed integral equation takes the form

$$
m^{*}\left(z^{*}\right)\left(\mathbf{I}-\mathbf{V}^{*}\right) q^{*}\left(z^{*}, t^{*}\right)+\mathbf{F}^{*} q^{*}\left(z^{*}, t^{*}\right)=\delta^{*}-g^{*}\left(z^{*}\right) .
$$

In this equation $\mathbf{I}$ is the identity operator.

Now the problem is to find the function $q^{*}\left(z^{*}, t^{*}\right)$ from equation (13).

\section{Solution of the dimensionless problem}

It should be noted that dimensionless functions $m^{*}\left(z^{*}\right)$ and $g^{*}\left(z^{*}\right)$ are related to the elastic properties of coating and shapes of the insert. They can vary rapidly changing. The presence of such a functions and integral operators of various types in main equation (13) does not allow the use of classical methods for solving this equation (Fourier, orthogonal polynomials, etc.). These methods does not take into account complex functions in the equation. This leads to significant errors in numerical calculations, in which it is necessary to restrict oneself to a finite number of members of an infinite series, which was constructed in a standard way without taking into account complex functions. Moreover, even if all these functions are constant, then the application of classical analytical methods will require solving an infinite system of Volterra integral equations, which leads to difficulties. Therefore, it is necessary to use a special approach to solve such a problem.

Note that the resulting integral equation in dimensionless form (13) coincides with equation (8) from work [4], provided that $P^{*}\left(t^{*}\right) \equiv 0$. Taking advantage of this, one can write out the final solution in dimensionless form

$$
\begin{gathered}
q^{*}\left(z^{*}, t^{*}\right)=\frac{1}{m^{*}\left(z^{*}\right)} \sum_{k=0}^{\infty} f_{k}\left(t^{*}\right) \sum_{m=0}^{\infty} \psi_{m}^{(k)} p_{m}\left(z^{*}\right), \\
-e^{-V^{*}\left(t^{*}-1\right)} \frac{g^{*}\left(z^{*}\right)}{m^{*}\left(z^{*}\right)}, \quad z^{*} \in[-1,1], \quad t^{*} \geq 1,
\end{gathered}
$$

where

$$
\begin{aligned}
& f_{k}(t)=\frac{g_{k}}{\gamma_{k}}\left\{e^{-V^{*}\left(t^{*}-1\right)}-\frac{1}{1+\gamma_{k}} \exp \left[-\frac{V^{*}\left(t^{*}-1\right)}{1+\gamma_{k}}\right]\right\}, \\
& g_{k}=\sum_{i=0}^{\infty} \psi_{i}^{(k)} \sum_{l=0}^{\infty} R_{i l} \int_{-1}^{l} \frac{p_{l}\left(\zeta^{*}\right) g^{*}\left(\zeta^{*}\right)}{m^{*}\left(\zeta^{*}\right)} d \zeta^{*} \text {, } \\
& R_{i l}=\int_{-1-1}^{1} \int_{1}^{1} \frac{k^{*}\left(z^{*}, \zeta^{*}\right) p_{i}\left(z^{*}\right) p_{l}\left(\zeta^{*}\right)}{m^{*}\left(z^{*}\right) m^{*}\left(\zeta^{*}\right)} d z^{*} d \zeta^{*}, \\
& J_{i}=\int_{-1}^{1} \frac{\left(\zeta^{*}\right)^{i}}{\sqrt{m^{*}\left(\zeta^{*}\right)}} d \zeta^{*}, \quad p_{0}\left(z^{*}\right)=\frac{1}{\sqrt{J_{0}}}, \\
& p_{j}\left(z^{*}\right)=\frac{1}{\sqrt{d_{j-1} d_{j}}}\left|\begin{array}{cccc}
J_{0} & J_{1} & \cdots & J_{j} \\
J_{1} & J_{2} & \cdots & J_{j+1} \\
\vdots & \vdots & \ddots & \vdots \\
1 & z^{*} & \cdots & \left(z^{*}\right)^{j}
\end{array}\right| \text {, } \\
& d_{i}=\left|\begin{array}{cccc}
J_{0} & J_{1} & \cdots & J_{i} \\
J_{1} & J_{2} & \cdots & J_{i+1} \\
\vdots & \vdots & \ddots & \vdots \\
J_{i} & J_{i+1} & \cdots & J_{2 i}
\end{array}\right| \\
& i, k, l=0,1,2, \ldots, \quad j=1,2,3, \ldots
\end{aligned}
$$

Here coefficients $\psi_{i}^{(k)}$ and $\gamma_{k}$ can be find from the solution of spectral problem

$$
\sum_{l=0}^{\infty} R_{i l} \psi_{l}^{(k)}=\gamma_{k} \psi_{i}^{(k)}, \quad i, k=0,1,2, \ldots
$$

\section{Solution of main problem}

Making the inverse change of variables according to formulas (12), one can obtain a dimensional solution of equation (11)

$$
\begin{gathered}
q(z, t)=\frac{a R(z)}{h_{\text {in }}} \sum_{k=0}^{\infty} f_{k}\left(\frac{t}{\tau_{0}}\right) \sum_{m=0}^{\infty} \psi_{m}^{(k)} p_{m}\left(\frac{z}{a}\right) \\
-\frac{\left[r_{\text {in }}-h_{\text {in }}-g(z)\right] R(z)}{h_{\text {in }}} \exp \left[-\frac{2 k_{\mathrm{w}} V\left(t-\tau_{0}\right)}{k_{\mathrm{H}} h_{\text {in }}}\right] .
\end{gathered}
$$

Consequently, contact stresses will be negligible over 
time. This is because the material in contact region wears out. Over time, the tension should disappear. However, it should be noted once again that the obtained solution can be used only for small values of wear. The solution obtained can be considered as the first step in the implementation of the numerical solution of problems with the amount of wear comparable to the thickness $h_{\text {in }}$ of the coating.

Having determined the contact pressure, it is possible to calculate the amount of coating wear according to formula (2).

\section{Conclusions}

Wear-contact problem for rigid insert, which diameter is variable, and elastic tube with complex inner coating is posed and solved. Analytical representation for contact stresses in interaction region is presented in series over special system of basis functions. This system depends on initial complex functions in main integral equation (nonuniformity and insert profile). It is shown that contact stresses will be negligible over time.

Contact rigidity $R(z)$ of the coating and insert form $g(z)$ are represented by separate factors and terms in final expression for contact stresses (17). This allows accurate calculations to be made, even if these parameters are described by rapidly changing functions. All other functions are quite smooth. This permits attaining a high accuracy by using only a few terms of the solution expansion even in the case of rapidly varying characteristics.

It is noted that the obtained solution can be used only for small values of wear. But can it be considered as the first step in the implementation of the numerical solution of problems with the amount of wear comparable to the coating thickness.

The present work was supported in part by the Ministry of Science and Higher Education of the Russian Federation in the framework of a Federal Target Program (contract No. AAAAA20-120011690132-4) and supported in part by the Russian Foundation for Basic Research (project No. 19-51-60001).

\section{References}

1. A.V. Manzhirov, K.E. Kazakov, Dokl. Phys. 62 (7), 344-349 (2017) DOI 10.1134/S1028335817070035

2. A.V. Manzhirov, K.E. Kazakov, AIP Conf. Proc. 1959, 070023 (2018) DOI 10.1063/1.5034698

3. K.E. Kazakov, S.P. Kurdina, J. Phys.: Conf. Ser. 1474, 012022 (2020) DOI 10.1088/17426596/1474/1/012022

4. K.E. Kazakov, S.P. Kurdina, IOP Conf. Ser.: Mater. Sci. Engng 904, 012010 (2020) DOI 10.1088/1757899x/904/1/012010

5. K.E. Kazakov, E3S Web Conf. 162, 02002 (2020) DOI 10.1051/e3sconf/202016202002

6. A.S. Pronikov, Wear and durability of machines (Mashgiz, Moscow, 1957) [in Russian]

7. M.M. Khrushchev, M.A. Babichev, Abrasive wear (Nauka, Moscow, 1970) [in Russian]

8. J. Collins, Failure of materials in mechanical design: analysis, prediction, prevention (Wiley, New York, 1993)

9. I.G. Goryacheva, M.N. Dobychin, Contact problems in tribology (Mashinostroenie, Moscow, 1988) [in Russian]

10. I.A. Soldatenkov, Wear contact problem with applications to engineering calculation of wear (Fizmatkniga, Moscow, 2010) [in Russian]

11. A.V. Manzhirov, V.A. Chernysh, J. Appl. Mech. Techn. Phys. 31, 894-900 (1990). DOI: 10.1007/BF00854204

12. N.Kh. Arutyunyan, A.V. Manzhirov, Contact problems in the theory of creep (Izd-vo Inst. Mekhaniki NAN Armenii, Yerevan, 1999) [in Russian]

13. J.F. Archard, J. Appl. Phys. 24 (8), 981-988 (1953) DOI 10.1063/1.1721448

14. A. Schalliamach, Proc. Phys. Soc. Sect. B 67 (12), 883-891 (1954) DOI 10.1088/0370-1301/67/12/304 